\title{
Asociación de Síndrome Nefrósico y Nefrocalcinosis Histopatológica
}

La nefrocalcinosis o depósito de calcio en el parenquima renal fue descrita por primera vez en 1934 por Albright como una complicación de un hiperparatiroidismo no tratado.

En 1935, Lighwood describe un caso necrópsico de nefrocalcinosis con paratiroides normales. En 1936, Butler notó ese fenómeno en niños con acidosis hiperclorémica. En 1947, Vaughan, Sosman y Kinney comunican el primer caso de nefrocalcinosis asociada a glomérulo nefritis crónica, haciendo hincapié èn su localización preferentemente cortical.

Posteriormente se han agregado otros casos, siendo 11 los publicados hasta la fecha, todos ellos en la literatura extranjera.

A continuación referiremos un caso de nefrocalcinosis asociado a glomerulopatía crónica nefrósica.

\section{CASO CLINICO}

La paciente M. E. S. F., historia clínica N. ${ }^{\circ} 677.084$, procedente de San Carlos, ingresa por primera vez a nuestro Servicio el 25 de febrero de 1974 a la edad de 11 años 6 meses con los diagnósticos de síndrome nefrósico y neumopatía izquierda.

Entre sus antecedentes familiares se consigna: madre 39 años, sana; padre 41 años, sano; 9 hermanos vivos, sanos. Ella es la $5 .^{\mathrm{a}}$ de sus hermanos. Es una familia de buenas condiciones socioeconómicas.

Su enfermedad actual se inicia en 1968 a los 6 años de edad. Ingresó al Hospital de San Carlos por presentar edema generalizado. Permaneció allí 3 meses, posteriormente fue trasladada a Santiago, donde permaneció hospitalizada 5 meses. Se le practicó biopsia renal obteniéndose sólo tejido medular. Fue dada de alta con

\footnotetext{
*Servicio y Depto. de Pediatría Hospital "Gmo. Grant Benavente".

**Departamento de Medicina. Escuela de Medicina Universidad de Concepción.
}

Dres. Rodriguez Y., Waldo*; Wilhelm P., Vera*; Reyes., Móniea*; Pomeroy F., John**.

el diagnóstico de síndrome nefrósico idiopático y con indicaciones de prednisona.

A los 45 días recae, siendo hospitalizada en Santiago por 3 meses. En esta oportunidad se efectuó estudio para lupus eritematoso, que fue negativo. No se repitió biopsia renal. Fue nuevamente dada de alta con prednisona.

Permaneció asintomática y con controles irregulares hasta 1972. Mientras continuaba con el tratamiento esteroidal presenta una peritonitis primaria, por lo cual fue intervenida en el Hospital de San Carlos por sospecharse una peritonitis apendicular. Fue dada de alta con el tratamiento adecuado de prednisona.

Permanece en buenas condiciones hasta febrero de 1974, en que nuevamente presenta edema generalizado, dificultad respiratoria y fiebre. Se hospitaliza en nuestro Servicio, donde se diagnostica una nueva recidiva de su síndrome nefrósico. La crisis nefrósica cedió al mes de terapia esteroidal y la neumopatía izquierda a la semana de iniciada la terapia antibiótica. En el momento del alta el 19 de abril de 1974, la uremia era normal y las proteinurias no eran significativas. Se intentó biopsia renal, que fue blanca en tres oportunidades. La pielografía descendente mostró nefromegalia bilateral con imágenes pielocaliciarias normales. ( $F_{0}-$ to N. ${ }^{\circ}$ la.)

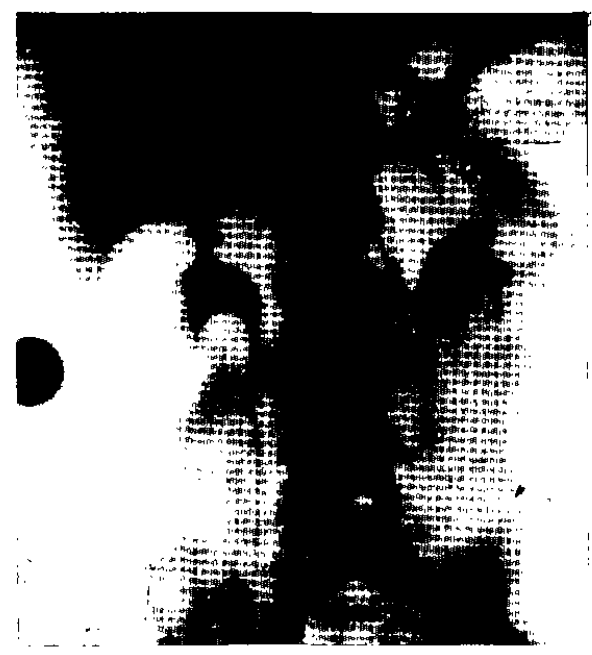


Posteriormente con controles ambulatorios y prednisona en Nefrología Infantil.

Se rehospitaliza el 7 de diciembre de 1974 y el 19 del mismo mes se practica biopsia renal, dándose de alta a las 48 horas. Después de su alta nos llega el resultado de la biopsia, que revela nefrocalcinosis con discreta lesión glomerular (Foto N. ${ }^{\circ}$ ). La niña no vuelve a control médico.

El 14 de febrero de 1976 a la edad de 13 años 6 meses, reingresa a nuestro Servicio con una nueva crisis nefrósica, peritonitis primaria, gran compromiso sensorial e insuficiencia renal aguda. A las 2 semanas se recupera totalmente de su I.R.A. y al mes remite su síndrome nefrósico.

Durante esta hospitalización se efectuó una biopsia renal, el 17 de marzo de 1976 , que reafirma el diagnóstico de nefrocalcinocis. (Foto N. ${ }^{\circ} 3$.)

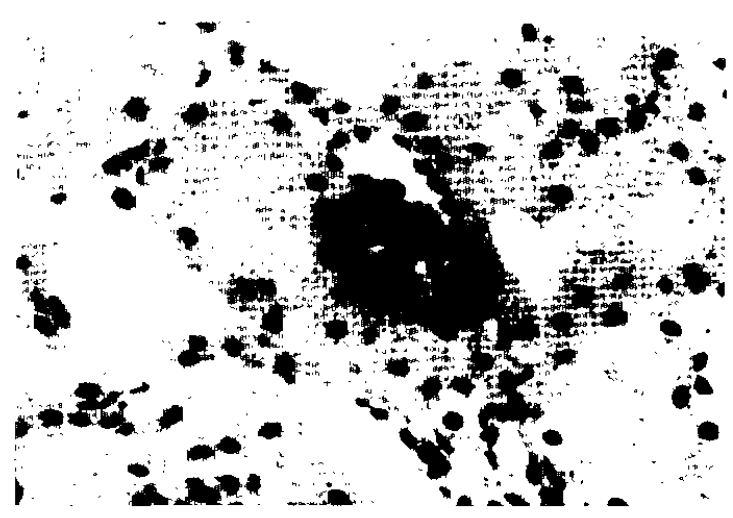

Vista panorámica de la biopsia percutánea. Al centro se ve en detalle este depósito de material calcáreo en un espacio que puede ser tubular o intersticial, que no está bien definido.

Reinterrogada la madre en forma dirigida señala categóricamente que ella por cuenta propia, administró mensualmente o cada dos meses una ampolla de vitamina $D$ de 600.000 U. entre los 2 y 4 años de edad, recibiendo la niña aproximadamente 20 dosis de golpes vitamínicos $\mathrm{D}$, en total.

Se planteo así la posibilidad de que la nefrocalcinosis fuera consecuencia y secuela de un cuadro de intoxicación por vitamina $\mathrm{D}$.

Sin embargo, en el diagnóstico diferencial era necesario descartar hiperparatiroidismo primario. Los resultados de las calcemias, fosfemias y fosfatasas alcalinas los hemos resumido en Tabla 1.

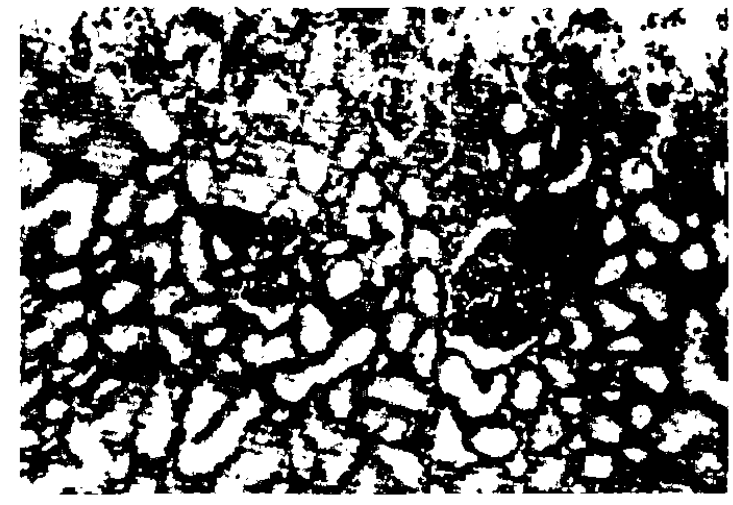

Al centro depósito calcáreo. A las 11 un glomérulo con aumento de la celularidad.

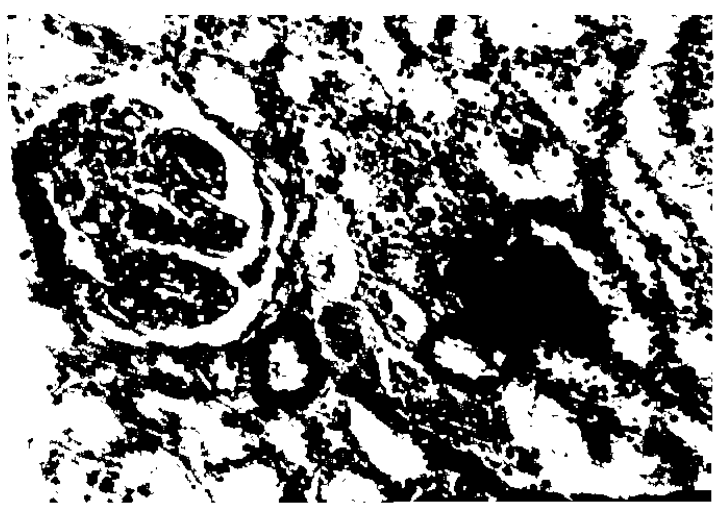

Glomérulo con mayor aumento, con material calcáreo en el polo vascular observándose leve ensanchamiento del mesangio. No hay fenomenos exudativos (granulocitos) ni proliferación del endotelio.

TABLAN. ${ }^{\circ} 1$

ESTUDIO DEL METABOLISMO Ca - P

\begin{tabular}{lccc}
\hline & $30-11 \mathrm{l}-76$ & $1-I V-76$ & $19-\mathrm{X}-76$ \\
\hline FOSFEMIA & $4.1 \mathrm{mg} \%$ & $4.05 \mathrm{mg} \%$ & $4.05 \mathrm{mg} \%$ \\
\hline CALCEMLA & $12.1 \mathrm{mg} \%$ & $11.4 \mathrm{mg} \%$ & $10.6 \mathrm{mg} \%$ \\
\hline CALCIURLA & $84 \mathrm{mg} / 24 \mathrm{hrs}$ & & \\
\hline FOSFATURL & $1.57 \mathrm{~g} \mathrm{P2} \mathrm{05}$ & & \\
\hline F. ALCALINAS & $5.16 \mathrm{U} \mathrm{B} \%$ & & $4.50 \mathrm{U} \mathrm{B} \%$ \\
\hline
\end{tabular}

TEST DE

SULKOWITCH $+\mathrm{a}++$ 


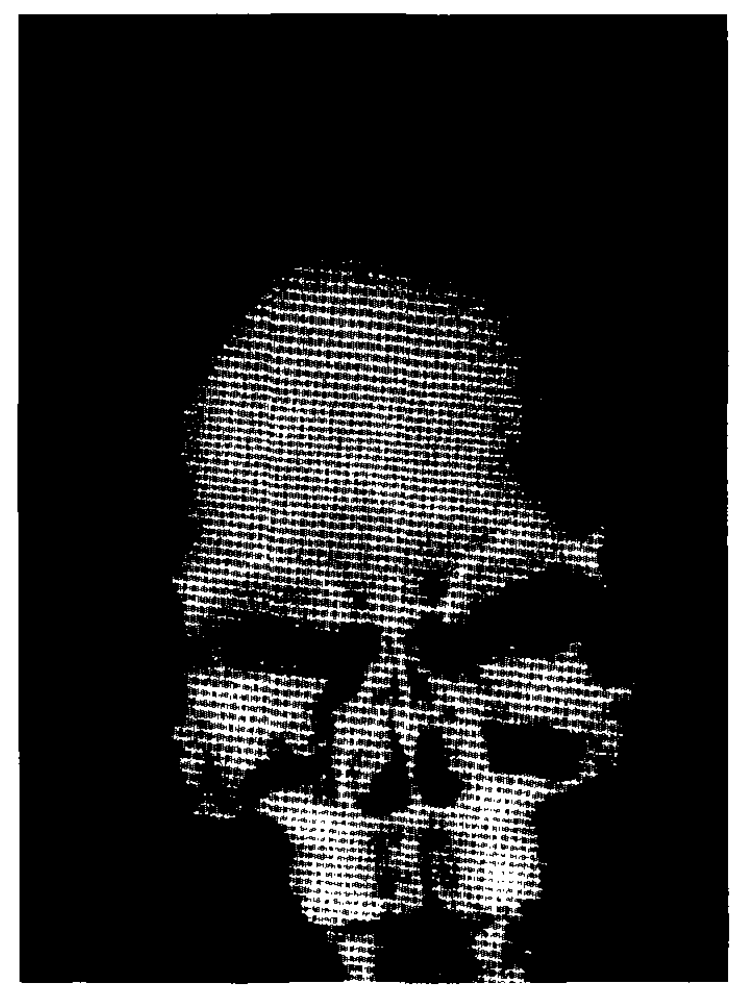

Foto 5

El estudio radiológico reveló ausencia de alteraciones óseas. (Fotos 5, 6, 7 y 8 )

Foto 7

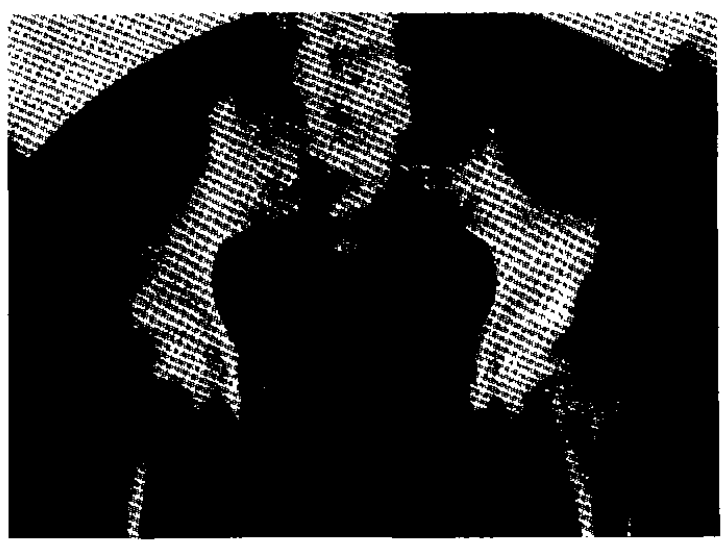

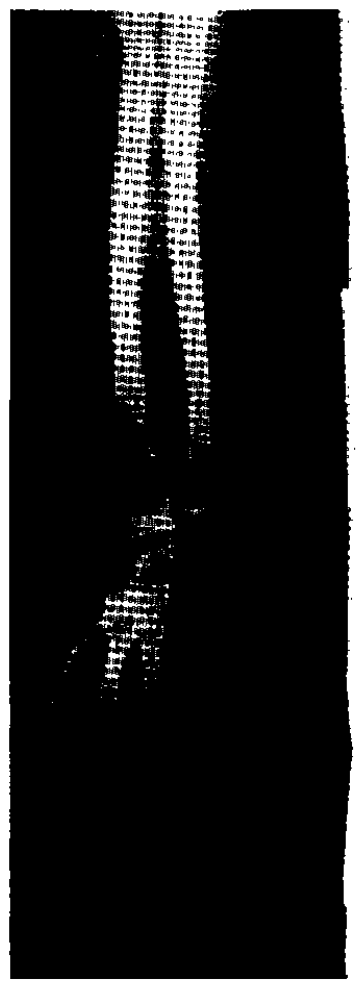

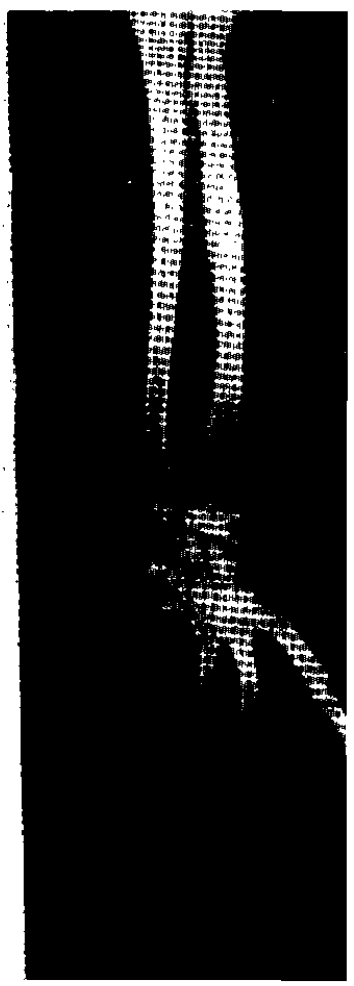

Foto 6

Foto 8

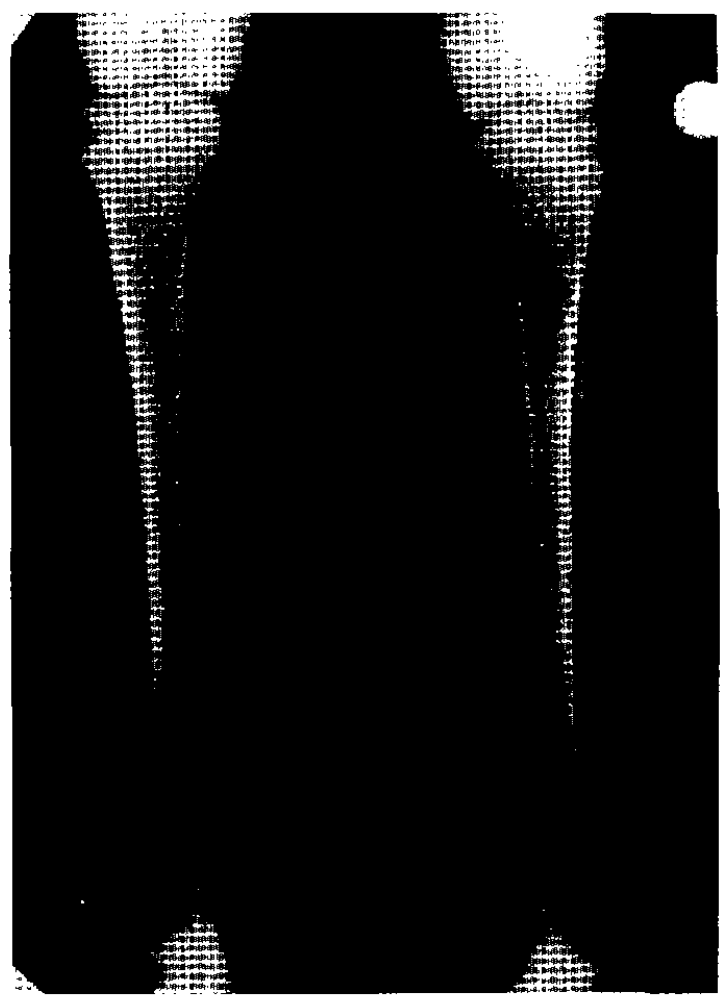


Estos elementos permitieron descartar un hiperparatiroidismo primario, reforzando como probable etiopatogenia la ingesta exagerada de vitamina $D$.

Fue dada de alta el 9 de abril de 1976 con los diagnósticos de: Glomenulopatía crónica, síndrome nefrósico en remisión, nefrocalcinosis.

Se ha controlado periódicamente en Policlínico de Nefrología Infantil. Permanece asintomática, en buenas condiciones, con su tratamiento de régimen hiposódico y prednisona. (Diap. Tabla N.o 2 .)

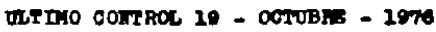

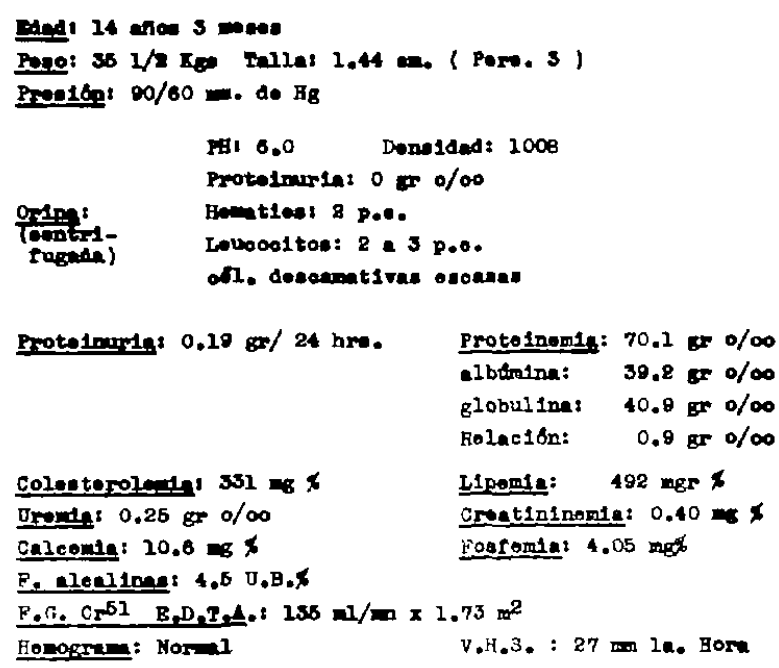

COMENTARIO

La hipercalcemia persistente está frecuentemente asociada a calcificaciones metastásicas, más frecuentemente observadas en riñones, pulmones, mucosa gástrica y vasos sanguíneos. Esta localización selectiva se cree que se deba a un factor fisiológico único.

El depósito de calcio en el riñón se lleva a cabo a nivel de los túbulos proximales comenzando en la membrana basal, para posteriormente en fases más avanzadas provocar un desprendimiento de las células tubulares, apareciendo el calcio como un depósito amorfo en la luz de lo que fue túbulo y penetrando de este modo al intersticio.
La etiopatogenia no está aclarada, se piensa que a nivel local sería la necrosis del parénquima lo que favorecería el depósito de calcio, mientras que a nivel general en casi todos los casos es constante el antecedente de ingesta exagerada de leche, vitamina $\mathrm{D}$, calcio, alcalinos o combinación de estos factores.

Seguramente debe existir algo más en la etiopatogenia que aún es desconocido para nosotros, probablemente un trastorno a nivel tubular, puesto que en las fases iniciales el calcio se localiza sobre los túbulos que morfológicamente son normales (diap. de etiopatogenia Tabla N. ${ }^{\circ}$ ).

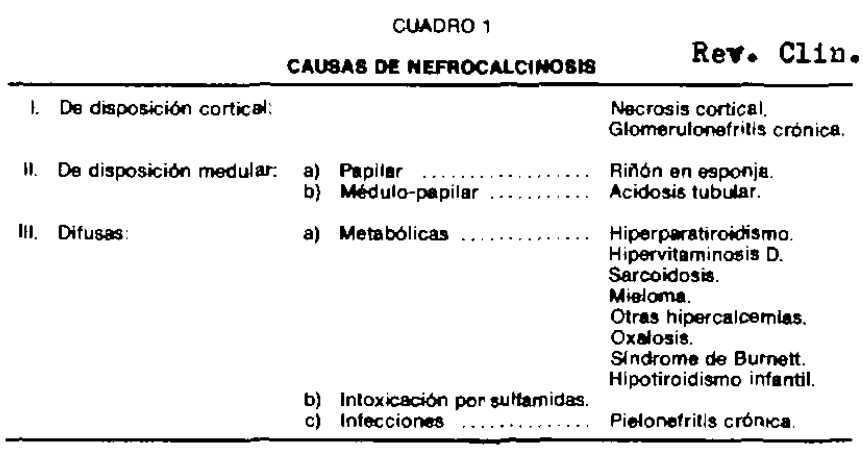


El diagnóstico en todos los casos ha sido un hallazgo fortuito radiologico, de biopsia o de necropsia. Su sintomatología estaría relacionada probablemente con los sintomas propios de hipercalcemia. Aproximadamente un $63 \%$ de estos niños presentan en algún momento de su evolución síntomas urológicas que pueden ser consecuencia de urolitiasis, infecciones urinarias, enuresis e incluso hematuria aislada.

El diagnóstico precoz, la corrección rápida de los desórdenes metabólicos, el tratamiento adecuado de las infecciones urinarias e inactivación de la causa, son básicas para un manejo correcto.

En nuestra revisión se concluye que con una función renal normal o cercana a lo normal existe aproximadamente un $55 \%$ de posibilidades de mejoría, pero aquellos niños con franca disminución de su función renal tienen un pobre pronóstico y evolucionan inexorablemente a una insuficiencia renal terminal y su sobrevida dependerá de la posibilidad de obtener un lugar en un programa de diálisis crónica o de un trasplante renal.

No existen diferencias de sexo y las edades de presentación fluctúan entre los 12 y 15 años, con un promedio de 8 años.

\section{RESUMEN}

Se presenta un caso clínico correspondiente a una glomerulopatía crónica, manifestada como síndrome nefrósico, asociado a nefrocalcinosis histopatológica.

Su etiopatogenia corresponde muy probablemente a ingesta exagerada de vitamina $D$.

Los exámenes de control practicados recientemente revelan una buena función renal y por lo tanto pensamos en un buen pronóstico hasta la fecha de $\mathrm{su}^{\circ}$ glomerulopatía y nefrocalcinosis asociada.

\section{REFERENCIAS}

1 Advani, U. H., Sidhu, G. S. Hypercalcemia and Unilateral Nephrocalcinosis. J.A.M.A. 232: 161-162, 1975.

2 Arones, W.I., Clurstensen, W. R. and Sosman, M.C. Specific Nephrocalcinosis of Glomerulonephritis Ann. Int. Med. 42: 260-282, 1955.

3 Esposito, W. J. Bilateral Diffuse Nephrocalcinosis Am. J. Roentg. Radium Ther. Nucl. Med. 101: 688-691, 1967.

4 Farrel, R. M., Horwith, M., Mueke, E. C. Renal tubular acidosis and Nephrocalcinosis: Diagnosis and clinical Management. J. Urol. 111: 429-433, 1974.

5 Geraci,J.E., Harris, H.W. and Keith N.M. Nephrocalcinosis visible by X-Ray associated chronic Glomerulonephritis. Mayor Clin. Proc. 25: 305-315, 1950.

6 Macias, A. y cols. Calcio ínnico y Litiasis Renal Cálcica Rev. Clin. Esp. 137: 455-458, 1975.

7 Malek, R.S.; Kelalis, P.P. Nephrocalcinosis in Infancy and childhood. J. Urol. 114: 441-443, 1975.

8 Miguel Alonso, J. L. y cols. Nefrocalcinosis asociada a glomerulonefritis cornica Rev. Clin. Esp. 137: 473-476, 1975 .

9 Moncrieff, M. W., and Chance, G. W. Nephrotoxic effect of vitamin D Therapy in vitamin D Refractory Rickets. Arch. Dis. Child. 44: 571-579, 1969.

10 Paunnier, L. et al. Renal Function and histology after Longterm vitamin D'Therapy of vitamin D Refractory Rickets J. Pediat 73: 833-844, 1968.

11 Roger, $P$. Nefrología Pediátrica, Ed. Toray S.A. 1. a edición, 1975.

12 Shanks, R. A., Mac Donald, A. M. Nephrocalcinosis Infantum. Arch. Dis. Child. 34: 115-119, 1952.

13 Skinner, D. G., Godwin, W. E. Indications for use of Intestinal Segments in management of Nephrocalcinosis, J. Urol. 113: 436-442, 1975.

14 Vásquez, A.M. Nephrocalcinosis and Hipertension in Juvenile Primary Hyperparathyroidism. Am. J. Dis. Child. 125: 104-106, 1973.

15 Wenzl, J. E. et al. Nephrolithiasis and Nephrocalcinosis J. Urol. 113: 436-442, 1975.

16 Woodard, J. C. A morphologie and Biochemical study of Nutritional Nephrocalcinosis in Female rats fed semipurified rats Am. J. Pathol. 65: 253-263, 1971. 